\title{
Kultur(en), Konflikt(e) und Unterricht(en)
}

\author{
Silke Ghobeyshi und Uwe Koreik
}

\section{Einführung}

Mit dem sogenannten interkulturellen Ansatz oder gar der interkulturellen Methode seit Mitte der achtziger Jahre hat das Thema Kultur einen hohen Stellenwert für den DaF-Unterricht - und dort nicht nur bei einer expliziten Landeskundevermittlung - bekommen (z. B. Müller-Jacquier 2001, CasperHehne 1999, ein Überblick über neueste Veröffentlichungen unter: www.ualberta.ca/ german / ejournal/anderswo.htm). Das Ziel des interkulturellen Ansatzes ist, um es sehr knapp zu formulieren: die Entwicklung von Handlungskompetenz für interkulturelle Kontaktsituationen auf der Basis einer geschulten Wahrnehmungs- und Aneignungsfähigkeit fremder Bedeutungen in unterschiedlichen verbalen und nonverbalen Zeichensystemen; dies mit dem Ziel, die Grenzen der eigenkulturell geprägten Wahrnehmung erkennen und überschreiten zu können, was $\mathrm{zu}$ einer erhöhten Empathiefähigkeit und Ambiguitätstoleranz führen soll. Dies nun wiederum hat zum Ziel, Mißverständnisse vermeiden bzw. erkennen $\mathrm{zu}$ können, und somit auf der Basis einer Komplementarität von erworbenem Wissen und einer erweiterten und geschulten Wahrnehmung Konflikte vermeiden bzw. besser mit ihnen umgehen zu können.

Nun sind Konflikte aber in z. T. erschrekkendem und auch hinsichtlich der $\mathrm{Zu}$ kunft beängstigendem Ausmaß in unse- rer Welt bereits gegeben, und der Titel des inzwischen zum Bestseller avancierten Buches von Huntington Kampf der Kulturen ist ein Thema, das von den Medien gern und oft komplexitätsbereinigt aufgegriffen wird. Davon zeugt beispielsweise auch der Artikel aus dem Wirtschaftsteil einer Zeitung, der das Scheitern von Unternehmensfusionen behandelt, das »Aufeinandertreffen von verschiedenen Kulturen " beklagt und der allen Ernstes mit "Kampf der Kulturen" überschrieben ist (Neue Westfälische vom 11.12.2002). Das Bild des konfliktreichen Aufeinanderprallens völlig unvereinbarer Mentalitäten begegnet uns allerorten und überlagert nicht selten eine tiefergehende politische und ökonomische Analyse der den Konflikten zugrundeliegenden tatsächlichen Ursachen.

Es wäre naiv anzunehmen, daß sich die zum großen Teil nicht nur das Individuum bedrohenden Konflikte in unserer Welt nicht auch latent oder auch offen in der DaF-Unterrichtssituation widerspiegeln könnten, auch wenn das Primat aller Sprachkursteilnehmer sicherlich das Erlernen der deutschen Sprache ist und nicht selten vielleicht auch das Bedürfnis überwiegt, mit den aus der Heimat mitgebrachten Problemen hier nun nichts zu tun haben zu wollen.

Der Fachverband Deutsch als Fremdsprache hat unter dem Einfluß des 11. September mit der Schaffung des Programmschwerpunktes "Spannungen in der Welt -Spannungen im Klassenzimmer« auf der 
FaDaF-Jahrestagung 2002 versucht, dem komplexen Problem Rechnung zu tragen, was in dem dazu veranstalteten Workshop auf große Resonanz stieß.

\section{Kulturen und Konflikte im DaF-Un- terricht}

Selbst wenn wir davon ausgehen, daß DaF-Lehrende durch ihre Ausbildung und ihre Berufspraxis über eine ausreichende interkulturelle Kompetenz verfügen, um dem oft mißbräuchlichen und fahrlässigen Umgang mit dem Begriff "Kampf der Kulturen " kritisch gegenüberzustehen, ist bei manchen Teilnehmern unserer Sprachkurse eine kritische Distanz solchen Simplifizierungen gegenüber nicht unbedingt $\mathrm{zu}$ erwarten. Erfahrungsgemäß trifft häufig eher das Gegenteil zu: Die mediale Aufbereitung von oft doch eher politischen Konflikten beeinflußt die Sprachkursteilnehmer in vielfältiger Weise. Durch die Tatsache, daß viele unserer Lernenden aus Ländern kommen, in denen die Massenmedien und hier insbesondere das Fernsehen nicht nur mit ethnischen und/oder kulturellen Stereotypen, sondern auch mit veritablen Feindbildern nicht anders als verantwortungslos umgehen, kann eine kognitiv geleitete kritisch-distanzierte Auseinandersetzung mit Gegenständen wie etwa der eigenen ethnozentrischen Wahrnehmung in der Unterrichtspraxis zu einer wahren Herausforderung werden. Vor dem Hintergrund der aktuellen politischen Weltlage scheint das Ziel "transnationale Kommunikationsfähigkeit« (Krumm 1994) noch ein Stück weiter in die Ferne gerückt zu sein.

Die konkreten Beispiele für diesbezügliche Konflikte im DaF-Unterricht aus der Fachliteratur oder unseren Befragungen (s. u.) sprechen für sich: Schroth-Wiechert (2001: 46) berichtet über ein sich aus der Unterrichtssituation ergebendes Streitgespräch zwischen russischen und litau- ischen Studierenden, welches die Lehrperson an die Grenzen der Möglichkeiten brachte, schlichtend bzw. überhaupt einzugreifen und sie es in Kauf nehmen mußte, daß andere Studierende sich abwandten bzw. den Raum verließen. Es wird von vehementen Auseinandersetzungen über den Israel-Palästina-Konflikt berichtet, bei denen es schwierig war, nach den kaum zu kontrollierenden Auseinandersetzungen wieder eine akzeptable Arbeitsatmosphäre im Kurs herzustellen. Und die zahlreichen uns bisher bekannt gewordenen Beispiele aus Unterrichtssituationen für Konflikte über Religion, die Rolle des Islam und unterschiedlich gelagerte Auseinandersetzungen über eine von vielen tatsächlich als unauflöslich wahrgenommene Kontroverse zwischen der arabischen und der westlichen Welt lassen erahnen, daß auch in der mittelfristigen Zukunft dieses Konfliktpotential weiterhin bestehen wird. Spannungen und Konflikte, die durch unterschiedliche »Wahrnehmungsstrukturen und Kommunikationsbedingungen" (Krumm 1994: 29) oder, etwas abstrakter, durch die Verletzung bzw. Infragestellung von selbst wahrgenommenen Selbstverständlichkeiten entstehen, bleiben in national heterogenen Gruppen, wie wir sie in den studienvorbereitenden und -begleitenden Deutschkursen an deutschen Hochschulen antreffen, nicht vor der Tür. Sie werden latent und manchmal auch offenkundig in den Klassenraum hereingetragen und könnten sich immer dann für eine Reflexion im DaF-Unterricht anbieten, wenn sie den Status des subjektiv Verborgenen verlassen, bergen aber andererseits genau das Konfliktpotential in sich, was von Lehrkräften häufig auch aus gutem Grund umgangen wird.

Konflikten, die durch vermeintliche kulturelle Unterschiede oder deren Konstruktion entstehen, liegen die drei folgenden Beobachtungen zu Grunde: 
1) Grundsätzlich kann sich an jedem Gegenstand ein Konflikt entzünden. Auftauchende unterschiedliche Überzeugungen und Einstellungen sind primär individuell $\mathrm{zu}$ verstehen, vor vorschnellen »kulturalistischen Konfliktdeutungen « (Auernheimer 1990) ist zu warnen.

2) Es gibt Konflikte, die durch die Konfrontation eigener, meist unbewußter und selbstverständlicher Deutungsund Handlungsmuster mit fremden, >unbekannten ' Deutungs- und Handlungsmustern entstehen. Konflikte dieser Art sind kulturbezogen.

3) Die Art der öffentlichen Auseinandersetzung und Desiderate in der Lehrendenausbildung $\mathrm{DaF}$ erschweren oder verhindern, daß kulturbezogene Konflikte von individuell, politisch oder ideologisch motivierten Konflikten adäquat unterschieden werden. Fähigkeiten, Strategien und Fertigkeiten im Umgang mit (Kultur-)Konflikten werden in der Lehrendenausbildung nicht oder nicht ausreichend vermittelt.

\section{3. »Kulturalität« im Fach DaF}

Anders als in der öffentlichen und medialen Auseinandersetzung mit 'Kulturkonflikten wird in der umfangreichen, teilweise höchst elaborierten und differenzierenden Fachliteratur die Einflußgröße Kultur präzise eingegrenzt. Während der gesellschaftliche Diskurs als $\mathrm{Zu}$ schreibungsprozeß bezeichnet werden kann, in dem Kultur als statische, situationsunabhängige und homogene Entität gehandelt und jeder relationale Charakter vergessen wird, gehört es etwa in der soziologischen oder der internationalen pädagogischen Forschung dazu, den je zugrundeliegenden Kulturbegriff zu konturieren und zu kontextualisieren. Die Forschungen im vergleichsweise immer noch jungen Fach Deutsch als Fremdsprache konzentrieren sich natur- gemäß zunächst auf die mit Sprache zusammenhängenden Manifestationen von Kultur, auf linguistischer Ebene insbesondere auf sprachkontrastive Grammatik, Semantik und Pragmatik, allgemein auf eine kontrastiv angelegte Erforschung und Vermittlung der in Sprache abgebildeten und transportierten kulturellen Selbstverständlichkeiten.

Daneben stehen seit den Kindheitstagen der Interkulturellen Germanistik die höchst abstrakt verhandelten Konzepte von Alois Wierlacher (»Das Eigene und das Fremde«) und Hans Hunfeld (»Doppelte Normalität des Fremden «), an die zu Recht die Frage gestellt werden kann, wie weit sie »uns tragen « können, im Deutsch-als-Fremdsprache-Unterricht und in der Lehrendenausbildung (Ehnert 1997: 46).

Von zentraler Bedeutung war und ist der Kulturbegriff auch in den oft selbstreferentiellen Debatten um Stellenwert und Funktion der Landeskunde. Hier hat sich

»(m)it dem Wandel von Landeskunde als einer reinen Institutionenkunde mit Ausflügen in die Hochkultur über oberflächliches ,Überlebenswissen ₹ zur Bewältigung des fremdkulturellen Alltags bis hin zu einem tieferen Verstehen der anderen Kultur(en) in einem deutschsprachigen Raum der Regionen (DACHL) [...] nun ein entscheidender Paradigmenwechsel vollzogen " (Fischer 2002: 92).

Auch wenn der Begriff des Paradigmenwechsels etwas zu hoch gegriffen sein sollte, gilt, daß dem Anspruch des Interkulturellen Lernens folgend die nunmehr verstehensorientierte (nicht rein kognitive!) Landeskunde die "Einbeziehung beider Kulturen" fordert (Fischer 2002: 93), die des Zielsprachenlandes gleichberechtigt neben der des Herkunftslandes (was folgerichtig die Einbeziehung aller vertretenen »Kulturen « in heterogenen Gruppen bedeutet). Vor dem Hintergrund der Tatsache, daß Kulturen und 
Gesellschaften viel zu komplex sind, »um - sei es aus der Innen-, sei es aus der Außenperspektive - gültig beschrieben werden zu können« (Ehnert 1997: 46), drängt sich hier einmal mehr die Frage nach dem Kulturbegriff im Fach Deutsch als Fremdsprache auf.

$\mathrm{Daß}$ in die Fachdiskussionen unterschiedliche Auffassungen von Kultur hineinwirken, versteht sich von selbst. Nicht selbstverständlich und unter Umständen irritierend ist jedoch, wie oft auf jegliche Konturierung des Begriffs verzichtet wird. Auch in der Auseinandersetzung mit dem Interkulturellen wird der Komplexität des kognitiven Konstrukts Kultur gerne aus dem Wege gegangen, scheint die Anwendung des Kulturbegriffs zu häufig beliebig zu sein.

Kultur ist wichtig, soviel steht fest, wenn z. B. Fischer (2002: 91) konstatiert, daß der »Umgang mit fremden Kulturen in und mittels fremder Sprachen « zu der »Kernkompetenz des global village schlechthin geworden« ist.

»Doch wie erkenne und beschreibe ich heute meine oder eine andere Kultur? Es hängt ja wieder mit der bedauernswerten Globalisierung zusammen, daß es zumindest oberflächlich betrachtet kaum mehr Kulturunterschiede gibt. Überall begegnen wir den gleichen Verhaltensweisen besonders Jugendlicher, Musik, Kleidung, Nahrung sind vereinheitlicht bis zum Überdruss. Unterschiede gibt es im wechselseitigen Hass, Unterschiede werden erkennbar, wenn man die Chance hat, dem einzelnen Kulturvertreter in seinem Alltagsleben zu begegnen, ihm genau zuzuhören, wenn man den Menschen auf die Finger, aufs >Maul<, auf den Körper schaut. Dann werden plötzlich Feinheiten kultureller Standards als Basis für Weltbewältigung sichtbar, die im Umgang mit dem Anderen sehr große Schäden anrichten können. (Ehnert 2002: 9-10).

Was Ehnert hier vorsichtig »Feinheiten kultureller Standards " nennt, kann vielleicht folgendermaßen interpretiert wer- den: Der von Alexander Thomas unseres Wissens nach im Deutschen eingeführte Begriff des Kulturstandards ist wohl nur verfeinert zu gebrauchen. Gerade DaFUnterricht und -Forschung, die dem Ansatz nach kulturkontrastiv angelegt sind, laufen leicht Gefahr, die gruppenspezifischen, > geteilten $<$ Standards im Wahrnehmen, Deuten und Handeln überzubewerten und die Wirksamkeit intersubjektiver und individueller Handlungs- und Bewertungsmodelle $\mathrm{zu}$ marginalisieren. Dennoch ist das Konzept der $>$ Kulturstandards deswegen nicht zu verwerfen. Das Ignorieren von Elementen, die als kulturbezogen einzuschätzen sind, ist ebenso kritikwürdig wie die Dichotomien eigen und fremd. Neuere (pädagogische) Ansätze, in denen Individuen nicht als Kulturvertreter, sondern als »Repräsentanten vielfältig zusammengesetzter Identitäten « (Ledoux, Leemann, Leiprecht 2001: 180) angesehen werden, übersehen womöglich, daß kulturbezogene Elemente für das Selbst selbstverständlich und unbewußt sind und daß die mannigfaltigen Unterschiede dieser unbewußten Selbstverständlichkeiten erst in sprach- und/oder kultur(!)kontrastiv angelegten Reflexionen $\mathrm{zu}$ Tage treten. Und wenn es das Ziel ist, »die Grenzen der eigenen ethnozentrischen Prägung zu überschreiten « (Krumm 1994: 31), sollte zunächst der Grenzverlauf geklärt werden.

\section{4. »Kulturthemen «}

In interkulturellen Kontaktsituationen und somit auch im Unterricht für Deutsch als Fremdsprache treten Konflikte auf, deren Genese sich allem Anschein nach von der Konfliktgenese in intrakulturellen Kontaktsituationen unterscheidet. Bereits >herkömmliche< Motivationsstörungen, wie sie in "westlichliberalen « Kursen nun einmal (sic!) vorkommen (Unaufmerksamkeit, explizites 
oder implizites Äußern von Unlust, das Ablenken von Mitschülern/Kommilitonen usw.) können innerhalb eines heterogen zusammengesetzten Kurses zu enormen Irritationen führen, die sich z. B. mit einer gänzlich anders regulierten gesellschaftshierarchischen Stellung des Lehrers in den Herkunftsländern erklären lassen. Das südkoreanische Sprichwort, demzufolge "students should not step on even a shadow of his or her teacher" (Weaver 1995: 23), spricht hier für sich. Solche unterschiedlichen kulturbezogenen Status- und Distanzregulierungen gehen einher mit anderen Vorstellungen von den Voraussetzungen und Realisierungen des (expliziten oder impliziten) Ausübens von Kritik, des (expliziten oder impliziten) Aussprechens von Lob und, nicht zu vergessen, des Sprechens oder des Schweigens über Tabuthemen. $\mathrm{Daß}$ die Stellung der Lehrenden ebenso wie die Lehr- und Lerngewohnheiten kulturspezifisch sind, ist bekannt, doch welche Konsequenzen hat dies für die Lernenden in unseren Kursen? In welchem Ausmaß die Unterrichtsatmosphäre durch die Nichtbeachtung kulturspezifischer Erwartungen gestört, ja vergiftet werden kann, sich auf die Lernmotivation und folgerichtig auch auf den Erfolg des Spracherwerbs auswirkt, wird u. a. von de Courcy (1997) beschrieben. Unseres Erachtens bedarf es hier neben weiterführenden unterrichtsevaluierenden Forschungsarbeiten einer fachspezifischen Auseinandersetzung mit dem Ziel der diesbezüglichen Qualitätssicherung des Unterrichts.

Das Kritisieren der Lehrerperson ist bei alledem nur ein Beispiel für Handlungen, die auszuüben für einige der Studierenden zumindest ungehörig und oft auch tabu sind. Daneben stehen eine Reihe von Themen, über die zu sprechen tabu ist. Als »tabuträchtige Bereiche" gelten z. B. Religion, Sexualität, Tod, Krankheit, Körper- funktionen, Eß- und Tischgewohnheiten und Aberglaube an die Bedeutung von Zahlen, Farben, Tieren (Schröder 1995, 1998). Daß auch historische Ereignisse tabuisiert werden können, darf in Deutschland als bekannt und internalisiert vorausgesetzt werden (Ghobeyshi 2002).

Tabus sind, einer Bezeichnung Schröders (1995: 16) folgend, »latente Kulturthemen«, d.h. sie gehören zu den oben erwähnten unbewußten Selbstverständlichkeiten. Es gibt mehr oder weniger stark ausgeprägte Tabus in jeder Gesellschaft, doch was tabuisiert wird, ist sowohl gruppen- und kulturbezogen als auch zeitspezifisch. Aufgrund des Veränderungsprozesses, dem Tabus unterliegen, kann es keinen "Katalog " geben (Schröder 1998: 197). Eine Vermittlung der dos and don'ts ist sehr hilfreich (s. die Aufnahme in Lehrwerken, z. B. Hansen/Zuber 1998), wird aber erst in einer durch offene Atmosphäre charakterisierten Unterrichtssituation geführten Diskussion weiterführend, denn »[e]rst im Kontrast der Kulturen, d.h. aus der Fremdperspektive bzw. im interkulturellen Kontakt, werden Tabus auffällig « (Schröder 1997: 105). Bezeichnend ist, daß Tabus für sentferntere Kulturen oder für die Vergangenheit als typisch angesehen werden, während die eigenkulturellen ebenso wie die individuellen Tabubereiche gar nicht bewußt sind und erst dann wahrgenommen werden, wenn sie gebrochen werden oder wenn nicht adäquat über sie kommuniziert wird (Schröder 1998: 197).

Vor dem Hintergrund, daß Tabuthemen am ehesten entsprochen wird, wenn man über sie schweigt, ergibt sich eine schwierige Situation für den Fremdsprachenunterricht. In interkulturellen Kontaktsituationen sind Tabuthemen und Tabubereiche des anderen häufig nicht nur unbewußt und unbekannt, sie sorgen bei ihrer Entdeckung während der Kommunika- 
tion oftmals für Befremden, Irritation und ein hohes Maß an Emotionalität. Auf die enorme Wichtigkeit der Sensibilität bei Themenwahl, Themenpräsentation und der methodisch-didaktischen Themenbehandlung im Unterricht kann daher gar nicht genug hingewiesen werden. Dabei darf keineswegs unberücksichtigt bleiben, daß »die Vorurteilsforschung inzwischen darauf aufmerksam gemacht hat, daß hartnäckige Klischees und Stereotype, die insbesondere bei der Beurteilung anderer Völker oftmals wirksam werden $[. .$.$] , nicht allein durch Informa-$ tion abgebaut werden können« (Ehnert/ Wazel 1994: 274).

Die von Krumm (1994: 31) vom Fremdsprachenunterricht geforderte $» F a ̈ h i g-$ keit, Verschiedenheit zu akzeptieren «, kann nur dann erfolgreich umgesetzt werden, wenn die verschiedenen Möglichkeiten der >Weltbewältigung < bewußt gemacht werden und eine Auseinandersetzung mit Unterschieden gezielt eingeübt wird, auch wenn sich dadurch Konfrontationen und Konflikte mitunter nicht vermeiden lassen. Für die Auseinandersetzung mit Tabuthemen fordert Schröder in diesem Sinne die

»Vermittlung von Euphemismen und anderen sprachlichen Strategien, die es dem Fremdsprachenlernenden ermöglichen, sich über tabuisierte Handlungen, Objekte, Sachverhalte und Wörter überhaupt verständigen zu können, ihn also dazu befähigen, sich auf der Mitte zwischen den beiden Polen eines völlig transparenten Diskurses und eines totalen Kommunikationsverbotes sprachlich souverän zu bewegen « (Schröder 1995: 33).

\section{Kulturbezogene Konflikte im DaF- Unterricht - eine Umfrage}

Um erste Hinweise auf die Konfliktthemen-Problematik aus Sicht der DaF-Lehrenden zu erhalten, haben wir im Herbst 2002 eine kleine Umfrage an verschiedenen deutschen Hochschulen durchge- führt (s. Fragebogen im Anhang). Aufgrund des zeitlich sehr eingegrenzten Untersuchungsrahmens sowie der begrenzten Streuung des Fragebogens kann der Rücklauf von 39 ausgefüllten Fragebögen in kurzer Zeit als hoch gewertet werden, wobei gleichzeitig klar ist, daß die Ergebnisse aufgrund der zu geringen Probandenzahl nicht als repräsentativ gelten können.

Es wurden Angaben zur Lehrperson statistisch erfaßt, quantifizierbare Daten erhoben sowie auch mangels vorangegangener Studien, die fundierte Kategorisierungen erlaubt hätten, verschiedene offene Fragen gestellt.

Ein erstes Ergebnis ist die Beobachtung (auch gespeist durch zahlreiche Rückmeldungen), daß etwas mehr als die Hälfte der Probanden den Fragebogen eher widerwillig, vielleicht aus Pflichtbewußtsein gegenüber den Autoren und teilweise mit Kritik an den (bewußt) nicht näher erklärten Begriffen >Konflikt ‘ und 'Kultur< ausgefüllt haben, und daß etwas mehr als ein Drittel den Fragebogen als eine interessante und spannende Herausforderung gesehen und z. T. in Kommentaren von über einer Seite Länge geantwortet hat.

Bei der Frage nach im Unterricht wahrgenommenen Konflikten, die nach Meinung der Lehrenden auf kulturelle Unterschiede zurückzuführen waren (Antwortspektrum von »nie « bis »sehr häufig«), fällt auf, daß die Mittelposition "mir nicht bewußt " nicht einmal angekreuzt wurde (weswegen sie aus der Auswertung herausgenommen wurde). Möglicherweise können Lehrende sich aufgrund ihres eigenen Anspruchs dies auch nicht eingestehen.

Weiter fällt auf, daß sich bei der Frage nach der Häufigkeit der beobachteten Konflikte zwei Gruppen herausbilden: 16 beobachten diese selten bis nie und 23 gelegentlich bis häufig (»nie« $=2$, »sehr häufig « $=0$ ). 
Der Versuch, Korrelationen zwischen diesen Antworttendenzen und Faktoren wie Geschlecht, Dauer der Berufspraxis, Lehrerfahrung in ausschließlich Hochschulen oder auch anderen Institutionen und gemachten und nicht gemachten Auslandserfahrungen herzustellen, führte zu keinem bzw. nur einem vagen Ergebnis. Es scheint sich lediglich anzudeuten, daß Lehrende mit mehr Lehrerfahrung (Mittelwert 13,64 Jahre) eher bereit sind, Konfliktthemen im Unterricht zu behandeln, als Lehrende mit weniger Lehrerfahrung (Mittelwert 8,24 Jahre). Angesichts der hohen Standardabweichungen muß aber auch diese Aussage mit Vorsicht betrachtet werden. Letztlich bleibt festzuhalten, $\mathrm{da} ß$ angesichts der mit einer Ausnahme sehr deutlich festzustellenden Streuung bei den Antworten die Hypothese naheliegt, daß auch bei einer erheblich größeren Untersuchungsgruppe hinsichtlich der Wahrnehmung von Konflikten und anderen Variablen keine Zusammenhänge bestehen.

Die am häufigsten genannten Themen, die Konflikte auslösten, sind: unterschiedliche Lerntraditionen und damit verknüpfte Erwartungen an die Rolle der Lehrenden, die Rolle der Frau sowie politisch brisante >Importthemen wie der Konflikt auf dem Balkan, der Israel-Palästina-Konflikt oder der Themenkomplex >Terror, Afghanistan und Irak«.

Bei der für uns wichtigen Frage, ob Lehrende bestimmte Themen im DaF-Unterricht $\mathrm{zu}$ vermeiden versuchen und den Gründen dafür, zeigt sich, daß etwa knapp ein Drittel hier dezidiert vorsichtig vorgeht (9 konkrete Einschränkungen und 2 allgemeine Einschränkungen) und etwa zwei Drittel sich offener verhalten (11 keine Einschränkungen und 15 keine Einschränkungen, allerdings mit methodisch-didaktischen und pädagogisch-psychologischen Hinweisen). Hinsichtlich der Frage nach eventuellen Korrelationen zwischen der Häufigkeit von erlebten Konflikten im Unterricht und der Bereitschaft, konfliktträchtige Themen im Unterricht $\mathrm{zu}$ behandeln, ergibt sich erneut kein Zusammenhang. Es deutet sich an, daß der Umgang mit Konflikten im Unterricht bei Lehrenden von subjektiven Einstellungen (sowie Erfahrungen?) und nicht von äußeren Faktoren abhängig ist. Bei einer näheren Analyse der Antworten der 15 Probanden, die zwar generell keine Themen vermeiden würden, dies allerdings von bestimmten Voraussetzungen abhängig machen, läßt sich für die Gesamtprobandengruppe feststellen, daß eine deutliche Mehrheit sich der Sensibilität und Problematik potentieller Konfliktthemen sehr bewußt ist und sie entweder $\mathrm{zu}$ vermeiden versucht oder deren Behandlung an intensiv reflektierte Bedingungen knüpft.

Wie sehr allerdings bei der Frage »Gibt es Themen, die Sie im DaF-Unterricht gezielt $\mathrm{zu}$ vermeiden versuchen? « die Positionen auseinandergehen, läßt sich an folgenden Zitaten verdeutlichen: "Nein, ich behandele gerade solche Themen", "Nein, es sollte grundsätzlich keine 'Schere im Kopf « geben « oder dem Hinweis darauf, daß sich ausländische Studierende auf eine offenere Diskurskultur in Deutschland einzustellen hätten und es deswegen auch aus politischen Gründen falsch sei, "wenn sich DaF-Lehrer/innen ständig selbst eine Zensur auferlegen würden, aus Angst vor einem (eventuellen) kulturbedingten Konflikt «. Demgegenüber steht exemplarisch folgende prägnante Aussage, nach der versucht wird, Themen aus folgenden Bereichen zu vermeiden: »Politik, Religion, Sexualität, Hunger, Folter, Elend, Krankheit, Tod und Gewalt. Vermieden werden soll die inhaltliche Kontroverse, wenngleich Vokabular und Idiomatik durchaus einmal vermittelt werden kann. Durch die Themen können einzelne KT [Kursteilnehmer, G./K.] unangenehm 
berührt werden. Wer will das denn auffangen? Sprachunterricht ist keine Therapieveranstaltung für Trauma- und Angstbewältigung. Er ist auch kein Agitationsforum.«

Bei der qualitativen Analyse der Antworten wird folgendes deutlich: Begründungen für die Vermeidung oder eingeschränkte Behandlung konfliktträchtiger Themen lassen Schwerpunkte erkennen. Dies sind die Sorge um das 'Kursklima oder die > Verletzung einzelner < sowie der gehäufte Hinweis auf die vom Thema abhängige fehlende eigene tiefgehende Kompetenz (z. B. bei den Themen >Islam<, >Israel-Palästina-Konflikt< oder >Religion<). Mehrfach wird die Besorgnis formuliert, daß ausgelöste Emotionen durch die Lehrenden nicht entsprechend aufgefangen werden können. Und nicht nur in diesem Zusammenhang wird häufig der Hinweis auf eine fehlende Ausbildung in diesem Bereich gegeben und werden entsprechende Verankerungen in den Lehrplänen oder gezielte Weiterbildungsmaßnahmen gefordert.

Bei dem Abgleich der Antworten zur Behandlung von Konfliktthemen im Unterricht mit dem Faktor Auslandserfahrung ist das Ergebnis allerdings auffällig: Diejenigen, die über Auslandserfahrung verfügen (22), teilen sich im Vergleich zur >reinen Inlandsgruppe worten mehrheitlich in zwei größere Gruppen, was bei einem Chi-Quadrattest eine zumindest tendenzielle Signifikanz ergibt. Zwei Pole sind erkennbar: die eine Gruppe der >Auslandserfahrenen ‘ behandelt Konfliktthemen, »wenn es sich so ergibt «, die andere »möglichst oft $«$. Alle Versuche, hier Korrelationen zu anderen Faktoren wie Berufserfahrung, Geschlecht, Länge des Auslandsaufenthalts oder auch des Aufenthalts in bestimmten Ländern bzw. Ländergruppen herzustellen, schlugen fehl. Auch hier bleibt nur die Hypothese, daß längere berufliche
Auslandsaufenthalte zwar das Verhalten der Lehrenden hinsichtlich des Umgangs mit Konfliktthemen beeinflussen, die Art und Weise des Einflusses aber offensichtlich stark von subjektiven Faktoren abhängig ist.

In einem ist sich aber die Gruppe der Befragten mehrheitlich deutlich einig: Die Behandlung von Konfliktthemen im DaF-Unterricht kann Veränderungen bewirken. Nur vier sind gegenteiliger Ansicht und ein weiterer vermutet zwar Veränderungen, aber eher zum Negativen: »Man ist genervt, fühlt sich indoktriniert. Es kann zur Reaktanz führen und zum Irrtum, in Deutschland müsse man sich immer kontrovers streiten." Offensichtlich gehen wir Lehrenden im Bereich $\mathrm{DaF}$ mit deutlicher Mehrheit - und ein ähnliches Ergebnis wäre bei einer erheblich ausgeweiteten Umfrage zu erwarten - davon aus, daß die Behandlung von Konflikt- und Tabuthemen Perspektiven erweitert, Relativierungen eigener (kulturbezogener) Standpunkte erlaubt, die Toleranz erhöht und damit die Bedingungen für ein friedliches Mit- bzw. Nebeneinander erhöht werden. Dies kann mit einem notwendigen Berufsoptimismus erklärt werden oder, negativ gewendet, mit einer spezifischen déformation professionelle; es kann aber auch als der Beleg für die vielfach gemachte - und unseres Wissens nach nicht erhobene - Erfahrung gesehen werden, daß sich im DaF-Unterricht auf neutralem Territorium positive Kontakte zwischen Angehörigen einander in z. T. sehr problematischen Kontexten verquickter Nationen und »Kulturen " entwickeln, die hoffnungsfroh stimmen können. Zu bedenken ist dabei, daß viele dieser Kursteilnehmer mit diesen differenzierten und positiven Erfahrungen in ihr Heimatland zurückkehren und dort relativ häufig in gesellschaftlich wichtigen Positionen, d.h. Mittlerfunktionen, einen Beruf ausüben. 


\section{Konfliktthemen im DaF-Unterricht}

Die wichtigste Voraussetzung für die Behandlung potentieller Konfliktthemen ist die Kompetenz der Lehrenden, wobei diese Kompetenz besonders dann auf dem Prüfstein steht, wenn es darum geht, Diskussionsverläufe richtig einzuschätzen und Diskussionen abzubrechen (welches u. U. ein gehöriges Maß an Autorität erfordert), bevor die Emotionen und Affekte nicht mehr zu kontrollieren sind. Die Auffassung, daß es unter 'gebildeten Leuten und demnach auch in der universitären Erwachsenenbildung eigentlich nicht zu unkontrollierbaren Gefühlsausbrüchen kommen dürfte, ist unserer Erfahrung nach und den zahlreichen Fallschilderungen in den Fragebögen zufolge unbegründet. Tatsächlich ist die $A f-$ fektbewältigung das zentrale Problem, denn bekanntlich entstehen Emotionen eben da, wo die eigenen - unter anderem kulturbezogenen - Grundwerte in Frage gestellt werden. So weist auch Hesse (2001) in seiner Beschreibung der »Aktualgenese interkultureller Konflikte « explizit darauf hin, daß in der "vehementen Phase « eines Konflikts jede der Parteien bestrebt ist, der »anderen Position die Verletzung eines höheren Wertes nachzuweisen« (ebd.: 155).

Um hier den eingangs aufgeführten Zielen des interkulturellen Ansatzes folgen und die Grenzen der eigenkulturell geprägten Wahrnehmung erkennen und überschreiten zu können, bedarf es daher vor allem des Wissens über die tatsächliche und konstruierte Entstehung von Konflikten (Konfliktgeschichte), über tatsächliche und konstruierte Konfliktzusammenhänge, über die Konfliktparteien, die Konfliktdynamik und über praktizierte Konfliktregelungen. Im Rahmen erprobter Methoden wie dem »fact-finding " (Jäger 1997) oder der »Themenzentrierten Interaktion « (Cohn 1980) kann eine inhaltliche Auseinandersetzung gelingen, in deren Verlauf nicht nur Konflikte analysiert und gehandhabt werden, sondern auch argumentatives Verhalten eingeübt werden kann. Voraussetzung ist auch hier, daß die Lehrenden auf die Konfliktthemen entsprechend vorbereitet sind, vor einer Auseinandersetzung nicht zurückscheuen und gleichzeitig aber die möglichen unter Umständen $\mathrm{zu}$ vermeidenden Konsequenzen im Blick haben.

Problematisch ist in diesem Zusammenhang die Gefahr, daß zukünftige DaFLehrende im Verlauf ihrer Ausbildung gar nicht oder nicht ausreichend darauf vorbereitet werden, auf komplexe und konfliktanfällige Themen adäquat zu reagieren bzw. reagieren zu können oder daß, wie Koreik (2001) beobachtet, zunehmend Defizite im »Weltwissen « der DaF-Studierenden festzustellen sind, was wiederum einen adäquaten Umgang mit potentiellen Konfliktthemen erschwert.

Wie problematisch und gleichzeitig wenig neu die Frage nach der Gratwanderung zwischen dem Aufgreifen von Konfliktthemen und deren Behandlung im DaF-Unterricht einerseits und der Gefahr, Probleme dadurch derart zu verstärken, daß normal ausgebildete DaF-Lehrkräfte ihnen nicht mehr gewachsen sind, andererseits ist, zeigt die dokumentierte und höchst interessante Diskussion nach einem Referat von Sabine Großkopf (1987) mit dem bezeichnenden Titel »Erfahrungen mit interkulturellen Lernsituationen zwischen Angst, Aggression und Lust «. Der Diskussionsteilnehmer Dausendschön (ebd.: 162) wirft die Frage auf, inwieweit weniger gut ausgebildete Lehrkräfte hinsichtlich der vorgestellten Unterrichtsmaterialien und -verfahren »Konflikte auslösen, Selbstreflexionen, Selbstanalysen bei Kursteilnehmern und Kursteilnehmerinnen in Gang setzen, selbst aber nicht über die therapeutischen Erfahrungen und Möglichkeiten verfügen, um diese dann auch aufzufangen«. 


\section{Schlußfolgerungen}

Sprachunterricht dient in erster Linie der Vermittlung von Sprachkompetenz. Nun ist es aber seit langem unbestritten, daß mit Sprachunterricht automatisch ein gewisses Maß an Kulturvermittlung einhergeht und auch einhergehen muß. Dabei ist bisher im wesentlichen - und dies $\mathrm{zu}$ Recht - die Zielkultur im Fokus aller Überlegungen. Gleichzeitig ist jedoch offensichtlich, daß vor dem Hintergrund einer zumeist national heterogenen $\mathrm{Zu}$ sammensetzung in den Sprachkursen an deutschen Hochschulen (und dies gilt selbstverständlich und möglicherweise erst recht auch für andere Institutionen) ganz andere kulturelle Differenzen den Unterricht maßgeblich beeinflussen (können). Themenorientierte Auseinandersetzungen mit auftauchenden Konflikten müssen verstärkt erprobt und evaluiert werden, und zwar nicht nur aus achtungs- und akzeptanzorientierten Gründen, die dem »friedlichen Zusammenleben der Menschen im Sinne der Charta der vereinten Nationen « (ABCDThesen 1990: 306) geschuldet sind, sondern auch sprachfunktional und sprachsystematisch als Übung zur Diskursfähigkeit, in deren Verlauf Argumentationslinien entwickelt werden können.

Die Umfrage unter Lehrenden legt den Schluß nahe, daß der Umgang mit Konflikten (vielleicht sogar schon deren Wahrnehmung) und vor allem die Bereitschaft, intensiver auf sie einzugehen, bei Lehrenden stark von subjektiven Einstellungen - und nicht etwa anderen Faktoren wie Berufserfahrungen, Geschlecht oder Unterricht in national homogenen oder heterogenen Gruppen - abhängig ist. Eine Konfliktbereitschaft ist dabei uneingeschränkt oder mit gewissen Einschränkungen - sehr hoch. Dabei dürfen, wie viele der Befragten auch hervorgehoben haben, die damit verbundenen Gefahren nicht unterschätzt werden. Sicher- lich ist dabei auch kritisch zu fragen, ob es im Kulturvergleich nicht vielleicht eher »typisch deutsch " ist, immer Probleme in den Vordergrund zu rücken, oder wie es ein Fragebogenteilnehmer formulierte, »aus kleinen Problemen gleich eine >Staatskrise< (zu machen), wo in anderen Ländern viel lockerer mit umgegangen wird? « Man darf auch in diesem Kontext die provokant formulierte Frage aufwerfen, "Soll am deutschen pädagogischen Wesen die Welt genesen?« (Klockow 1990), sollte dabei aber nicht übersehen, daß die fachwissenschaftliche Thematisierung von Kulturkonflikten im Unterricht nun wirklich keine deutsche Erfindung ist.

Die Bundesrepublik Deutschland strebt nicht nur eine Erhöhung des Anteils ausländischer Studierender an, sondern ist aufgrund der demographischen Entwicklung zunehmend darauf angewiesen, hochqualifizierte ausländische Hochschulabsolventen in die eigene Gesellschaft zu integrieren. Dabei kann es nur von Vorteil sein, wenn diese zukünftigen Mitbürger eine Diskussionskultur erlebt und erlernt haben, wie sie in entsprechenden gesellschaftlichen Kreisen der deutschen Gesellschaft gepflegt wird. Es ist also tatsächlich eine Verbesserung der interkulturellen Kompetenz auf Seiten der Lehrenden wie Lernenden anzustreben, wobei gleichzeitig folgendes offensichtlich ist:

»In Zeiten der Zertifizierung, der Portfolios und der Qualitätssicherung fehlt zur Zeit noch völlig ein Instrumentarium zur Messbarkeit (inter)kultureller Kompetenz." (Fischer 2002: 94)

Wir sind uns bewußt, daß wir letztlich mehr Fragen aufgeworfen als Antworten gegeben haben. Dies nehmen wir aber in Kauf, da wir zu wissen glauben bzw. spüren, daß zumindest einige der Fragen tatsächlich dringend der Antwort bedürfen. 


\section{Literatur}

»ABCD-Thesen zur Rolle der Landeskunde im Deutschunterricht «, Deutsch als Fremdsprache 2 (1990), 303-308.

Aguado, Karin; Riemer, Claudia (Hrsg.): Wege und Ziele: zur Theorie, Empirie und Praxis des Deutschen als Fremdsprache (und anderer Fremdsprachen). Festschrift für Gert Henrici zum 60. Geburtstag. Baltmannsweiler: Schneider Verlag Hohengehren, 2002.

Auernheimer, Georg: Einführung in die interkulturelle Erziehung. Darmstadt: Wissenschaftliche Buchgesellschaft, 1990.

Auernheimer, Georg; van Dick, Rolf; Petzel, Thomas; Wagner, Ulrich (Hrsg.): Interkulturalität im Arbeitsfeld Schule. Opladen: Leske + Budrich, 2001.

Casper-Hehne, Hiltraud: »Interkulturelle Kommunikation. Neue Perspektiven und alte Einsichten «, Zeitschrift für Angezwandte Linguistik (ZfAL) 31 (1999), 77-107.

Cohn, Ruth: Von der Psychoanalyse zur themenzentrierten Interaktion. Stuttgart: Klett, 1980.

de Courcy, Michèle: »Teaching and learning with different scripts: Cross-cultural conflict in a chinese late immersion classroom «, Language and Education 11,4 (1997), 242-259.

Ehnert, Rolf: »Neuorientierungen für das Ausbildungsfach Deutsch als Fremdsprache«, Germanistische Linguistik 137-138 (1997), 43-53.

Ehnert, Rolf: »Einige Gedanken zur Lehrerausbildung für das Fach Deutsch als Fremdsprache", Mitteilungsblatt des Landesverbands Westfalen-Lippe des Fachverbands Moderne Fremdsprachen 20,2 (2002), 7-12.

Ehnert, Rolf; Wazel, Gerhard: »Landeskunde«. In: Henrici, Gert; Riemer, Claudia (Hrsg.): Einführung in die Didaktik des Unterrichts Deutsch als Fremdsprache. Baltmannsweiler: Schneider Verlag Hohengehren, 1994, 273-281.

Fischer, Roland: »Die Theorie ist's und nicht die Praxis«, Bulletin suisse de linguistique appliquée 75 (2002), 91-95.

Ghobeyshi, Silke: Nationalsozialismus und Schoah als landeskundliche Themen im DaFUnterricht. Frankfurt a. M.: Lang, 2002 (Werkstattreihe Deutsch als Fremdsprache, 72).

Großkopf, Sabine: »Erfahrungen mit interkulturellen Lernsituationen zwischen Angst, Aggression und Lust «. In: Gerig- hausen, Josef; Seel, Peter C. (Hrsg.): Aspekte einer interkulturellen Didaktik. Dokumentation eines Werkstattgesprächs des GoetheInstituts München vom 16.-17. Juni. München: Goethe-Institut, 1987, 134-169.

Hansen, Margarete; Zuber, Barbara: Zwischen den Kulturen: Strategien und Aktivitäten für landeskundliches Lehren und Lernen. Berlin u. a.: Langenscheidt, 1998.

Helbig, Gerhard; Götze, Lutz; Henrici, Gert; Krumm, Hans-Jürgen (Hrsg.): Deutsch als Fremdsprache. Ein internationales Handbuch. 2. Teilband (Handbücher zur Sprach- und Kommunikationswissenschaft, 19.2). Berlin; New York: de Gruyter, 2001.

Hesse, Hermann-Günther: "Zur Aktualgenese interkultureller Konflikte«. In: Auernheimer, Georg et al. (Hrsg.), 2001, 141160.

Jäger, Uli: Soft Power. Wege ziviler Konfliktbearbeitung. Tübingen 1997.

Klockow, Reinhard: "Soll am deutschen pädagogischen Wesen die Welt genesen?«, Info DaF 17,1 (1990), 64-68.

Knapp-Potthoff, Annelie; Liedke, Martina: Aspekte interkultureller Kommunikationsfähigkeit. München: iudicium, 1997.

Koreik, Uwe: „Große Töne, wenig Musik. Zur Rolle der Landeskunde im Fach Deutsch als Fremdsprache«. In: Aguado, Karin; Riemer, Claudia (Hrsg.) 2001, 3949.

Krumm, Hans-Jürgen: »Mehrsprachigkeit und interkulturelles Lernen. Orientierungen im Fach Deutsch als Fremdsprache", Jahrbuch Deutsch als Fremdsprache 20 (1994), 13-36.

Ledoux, Guuske; Leemann, Yvonne; Leiprecht, Rudolf: »Von kulturalistischen zu pluriformen Ansätzen. Ergebnisse des niederländischen Forschungsprojekts $>$ Interkulturelles Lernen in der Klasse « In: Auernheimer, Georg et al. (Hrsg.) 2001, 177-195.

Müller-Jacquier, Bernd-Dietrich: »Interkulturelle Landeskunde«. In: Helbig, Gerhard; Götze, Lutz; Henrici, Gert; Krumm, Hans-Jürgen (Hrsg.) 2001, 1230-1234.

Schröder, Hartmut: »Tabuforschung als Aufgabe interkultureller Germanistik «, Jahrbuch Deutsch als Fremdsprache 21 (1995), 15-35.

Schröder, Hartmut: »Tabus, interkulturelle Kommunikation und Fremdsprachenun- 
terricht. Überlegungen zur Relevanz der Tabuforschung für die Fremdsprachendidaktik«. In: Knapp-Potthoff, Annelie; Liedke, Martina (Hrsg.) 1997, 93-106.

Schröder, Hartmut: »Interkulturelle Tabuforschung und Deutsch als Fremdsprache«, Deutsch als Fremdsprache 35 (1998), 195-198.

Schroth-Wiechert, Sigrun: Deutsch-alsFremdspracheunterricht ohne Lehrwerk für heterogene LernerInnengruppen im Zielsprachenland unter besonderer Berïcksichtigung des interkulturellen Ansatzes. Frankfurt a. M.: Lang, 2001 (Werkstattreihe Deutsch als Fremdsprache, 70).

Weaver, Gary R.: "Communication and Conflict in the multicultural classroom «, Adult Learning 6,5 (1995), 23-24.

(www.ualberta.ca/ german/ejournal/anderswo.htm)

\section{Anhang}

Fragebogen:

Kulturbedingte Konfliktthemen im Klassenraum (DaF-Unterricht)

Angaben zur Lehrperson:

$\square$ weiblich DaF-Unterrichtspraxis seit Jahr(en)

$\square$ männlich

DaF-Unterrichtspraxis

$\square$ an der Hochschule:

außerhalb der Hochschule:

bei der VHS

$\square$ sonstigen freien Kursanbietern, nämlich

im Ausland $\square$ nein $\square$ ja

Wenn ja: in

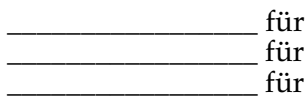

$\begin{array}{ll}\text { für } & \text { Monat(e) } \\ \text { für } & \text { Monat(e) } \\ \text { für } & \text { Monat(e) }\end{array}$

Haben Sie auch national homogene Gruppen unterrichtet?

Wenn ja, welche?

Bitte kennzeichnen Sie bei den folgenden Fragen, wenn es sich um Erfahrungen in national/kulturell homogenen Gruppen handelte.

1. In den von mir geleiteten DaF-Kursen tauch(t)en Konflikte auf, von denen ich meine, daß sie auf kulturelle Unterschiede zurückzuführen sind.

\begin{tabular}{|l|l|l|l|l|l|l|}
\hline nie & sehr selten & selten & mir nicht bewußt & gelegentlich & häufig & sehr häufig \\
\hline
\end{tabular}

* bitte ankreuzen

Wenn kulturbedingte Konflikte auftauch(t)en, betrafen sie:

$\square$ Studierende und mich $\square$ Studierende und andere Kursteilnehmer $\square$ alle im Kurs

Solche Konflikte beruhten auf folgenden Anlässen bzw. Themen: 
Wenn Sie selbst (mit) betroffen waren, warum?

bitte ggf. die Rückseite nutzen

2. Sollten im DaF-Unterricht gezielt Themen behandelt werden, die auch zu einer konfliktreichen Diskussion führen könnten? \begin{tabular}{|l|l|l|l|l|}
\hline auf keinen Fall & möglichst selten & wenn es sich so ergibt & gelegentlich & möglichst oft \\
\hline
\end{tabular}

Warum haben Sie so entschieden?

bitte ggf. die Rückseite nutzen

3. Kann sich Ihrer Meinung nach durch die Behandlung von Konfliktthemen im DaFUnterricht etwas ändern? $\square$ ja $\square$ nein

Wenn ja, was?

bitte ggf. die Rückseite nutzen

4. Gibt es Themen, die Sie im DaF-Unterricht gezielt zu vermeiden versuchen?

Wenn ja, welche und warum? (z. B. politische, religiöse, Sexualität u. a.)

bitte ggf. die Rückseite nutzen 